\title{
versants
}

\section{Gilliard et famille}

\author{
Daniel MAgGeTtI \\ Université de Lausanne \\ ORCID: 00oo-0003-I285-7872
}

\begin{abstract}
Les nombreuses facettes des activités intellectuelles et littéraires de Henri Roorda ont laissé peu de traces manuscrites ou documentaires. Parmi les rares lettres de l'écrivain accessibles dans des archives, celles adressées à Edmond Gilliard et à la mère de ce dernier témoignent d'une proximité qui, toute discrète qu'elle est, n'en demeure pas moins exceptionnelle pour Roorda dans le contexte artistique lausannois. L'amitié que Gilliard manifestera à Roorda notamment dans son texte d'hommage posthume semble avoir été particulièrement importante pour la veuve de ce dernier, si l'on en juge par les quelques billets de sa main dont l'auteur du Pouvoir des Vaudois et son épouse sont les destinataires.
\end{abstract}

Keywords : Henri Roorda, Lili Roorda, correspondance, Edmond Gilliard, Ernest Ansermet, Marie Gilliard-Malherbe, Alice Gilliard, C. F. Ramuz, Cahiers vaudois.

Le nom de Henri Roorda n'est pas de ceux qui se rencontrent souvent dans les inventaires des fonds d'archives. Très peus de manuscrits de l'écrivain ont été conservés; si lui-même semble avoir détruit, avant son suicide, la correspondance qu'il avait reçue, les lettres qu'il a adressées à ses relations sont elles aussi en petit nombre, et ne permettent nullement ne fût-ce que d'esquisser le réseau amical et intellectuel au sein duquel il a évolué. D'où l'intérêt particulier que revêtent les quelques missives de Roorda destinées à Edmond Gilliard (I875-1969) et à la mère de ce dernier : ce sont là les rares traces d'une sociabilité dont on mesure qu'elle n'est pas synonyme de familiarité, mais qui laisse transparaître une forme de proximité confiante sans doute exceptionnelle pour l'écrivain.

Dans l'histoire littéraire, les noms de Roorda et de Gilliard ont pour commun dénominateur les Cahiers vaudois, l'entreprise mi-revue mi-maison d'édition cofondée par Gilliard et Paul Budry en 19I4, qui publie en I9I7 Le Pédagogue n'aime pas les enfants. Mais le caractère d'exclusivité de leur relation prend racine dans le contexte du Gymnase cantonal : selon les propres dires de Gilliard en 1942 dans L'École contre la vie, c'est là qu'il a apprécié, chez deux seuls de ses collègues, Roorda et Ernest Ansermet, le fait qu'ils "parlaient avec une naturelle aisance "humaine" », en étant "vraiment capables de parole "mondaine", et nets de toute verbosité scolaire» (Gilliard I965: 429). Dans À Henri Roorda, son texte d'hommage paru en I929, Gilliard souligne à la fois les profondes différences qui le séparent de son ami dispa$\mathrm{ru}$, et la convergence de leur position et de leurs constats : 
C'est par des procédés de pensée bien différents que nous sommes arrivés à des certitudes semblables [...] ; des logiques en apparence opposées ont abouti à une même résolution morale, nous ont imposé une même attitude civile. [...]

Notre amitié est d'autant plus significative que nos tempéraments, nos constitutions naturelles, nos complexions physiques; somme toute, notre timbre et notre trempe ; notre allure, nos inclinations simples et nos goûts privés ; - notre taille, notre coupe, notre individuelle dégaine - rien de tout cela ne s'accordait de soi-même ; tout se fût contrarié, à le prendre dans l'usage commun, et à l'estimer au cours vulgaire de la vie (Gilliard 1965 : 374).

Aux yeux de Gilliard, l'étranger "du dehors" qu'est Roorda communie avec lui-même, étranger "du dedans", par le partage d'une forme de solitude existentielle et de décalage social. Sous le ton volontiers lyrique de l'évocation, l'on devine ce que les lettres mettent également en évidence, à savoir la pudeur et la discrétion qui caractérisent la camaraderie entre les deux hommes. Les lettres de Roorda rassemblées ci-après (les réponses, forcément, n'existent plus) révèlent simultanément une fidélité jamais démentie, et une fréquentation ne débordant guère le contexte professionnel. Cette impression est confirmée par les billets que Lili Roorda, devenue veuve, adresse à Gilliard : c'est par les récits de son mari ou parce qu'elle a assisté à ses conférences qu'elle le connaît alors, non pour l'avoir rencontré en famille ou dans un cadre de sociabilité commune'.

Étant donné la rareté de traces épistolaires de Roorda, les documents que nous reproduisons constituent des jalons précieux pour mieux cerner la personnalité de l'écrivain, et pour compléter la connaissance de sa réception posthume, y compris au sein de son cercle familial. Anodins parfois, ces échanges ne sont dès lors jamais anecdotiques pour le lecteur du XxI siècle.

I À ces liens on peut ajouter les relations entre Gertrude Schroeder-Roorda van Eysinga (I877-I960), la demi-sœur de l'écrivain, et Gilliard, attestée par des traces épistolaires; elles semblent cependant s'inscrire dans le contexte des réseaux théosophiques, et sans rapport avec l'œuvre de Roorda ou sa réception. 


\title{
LETTRES
}

\section{Henri Roorda à Edmond Gilliard}

\author{
Les Diablerets ${ }^{2}$ \\ Vendredi, 28 [juillet I9II]
}

\section{Cher ami,}

Je suis ici depuis deux jours et j'en partirai peut-être dimanche. Je dois faire avec Mayor ${ }^{3}$ une course de 3, 4 ou 5 jours. Il va m'écrire si c'est pour la semaine qui vient ou pour plus tard. La perspective d'aller causer avec toi me réjouit; mais, avant la réponse de l'autre, je ne puis pas te proposer une date précise. Quand je serai revenu, n'importe quel jour que tu voudras bien me proposer me conviendra. Si notre excursion est retardée, je t'avertirai aussitôt.

Ansermet vient de recevoir ta lettre. Il revenait du Scex Rouge ${ }^{4}$ dont il avait fait la dangereuse ascension; et, après une absinthe légère et un tub énergique, il s'est couché !

Madame, confuse de son trop long silence, t'envoie des amitiés cordiales et te fait savoir qu'ils auront, la semaine prochaine, la visite de Ramuz'. La date est fixée car c'est en se rendant au Valais (si je me souviens bien) qu'il s'arrête aux Diablerets. Après son départ, la voie sera libre et on t'appellera aussitôt pour un séjour aussi long que possible.

J'ai été vivement impressionné par ton billet de l'autre jour et par celui que tu écris à $\mathrm{A}$ [nsermet]. Les quelques mots qui accompagnaient le billet de 300 frs. sont exquis ${ }^{6}$. Mais c'est sur autre chose que je voudrais insister. Je me dis qu'il est très bon que tu aies appris la décision irrévocable six semaines

2 Comme on le voit plus loin, Roorda est l'hôte d'Ernest Ansermet dans cette localité des Alpes vaudoises.

3 Vraisemblablement Benjamin Mayor (I866-1936), ingénieur, professeur de mécanique et de physique à l'université de Lausanne.

4 Le Scex Rouge est un sommet du massif des Diablerets, culminant à 297I mètres.

5 C. F. Ramuz séjournera aux Diablerets chez les Ansermet, dans leur chalet « La Retraite», du I9 au 28 août (Ramuz 2005 : 205). Le romancier connaît de longue date, par sa famille, l'épouse d'Ansermet, née Marguerite Jaccottet (I875-1940), avec qui il correspond également. 6 Roorda fait allusion à "l'affaire du Gymnase" : en I9I0-I9II, Gilliard a remplacé avec succès, au Gymnase classique, Abel Biaudet, qui a démissionné en I9II. Pour lui succéder, on a nommé, sans concours, le romancier populaire Benjamin Vallotton. Gilliard, qui espérait obtenir cette place, a reçu comme indemnité du Département de l'instruction publique, pour les éventuels achats de livres qu'il aurait faits, un bon de trois cents francs, qu'il a retourné immédiatement tout en donnant sa démission de maître de classe au Collège cantonal. 
avant la rentrée. Si tu avais su la chose au moment de reprendre tes leçons, ta besogne t'aurait sans doute profondément dégoûté7.

Tu as quelques semaines devant toi. Profites-en pour songer un peu à tes premières leçons, afin d'éviter le désarroi, l'hésitation et le découragement des premiers jours. Profites-en surtout pour faire un beau projet, pour mettre un travail en train. Profites-en pour accepter ce qu'il y a d'inévitable dans la situation, tout en songeant à une belle revanche possible. Tu dis que tu sors de la Commission dont tu faisais partie. Mais n'aies pas un nouveau mouvement d'humeur. À quoi bon ? De deux choses l'une : ou bien tu restes dans la boîte pédagogique et dans ce cas il faut essayer d'y être le moins mal possible et d'éviter les conflits dont EUX ne souffriraient absolument pas (question d'épiderme). Ou bien tu t'en vas, et alors il s'agit de bien savoir où tu iras.

Évidemment, celui qui te dit cela est un père de famille dépourvu de rentes et qui tient à son modeste confort et à son vin quotidien. Et il te le dit bien mal. Si nous causions ensemble, je m'exprimerais plus clairement. Ici, dans l'atmosphère endormante de cet après-midi, mon style est d'une matité qui m'afflige. En tous cas, je me répète, qu'un travail passionnant qu'on vient de commencer peut être un excellent remède. Peut-être qu'à toi une besogne de paysan ou de jardinier fera plus de bien encore. Je n'en sais rien. Et l'un n'empêche pas l'autre. Quoi qu'il en soit, arrive promptement par le dédain à la sérénité ! Reclus ${ }^{8}$ me disait parfois : "Qui se contient, s'accroît. »

Je suis mécontent de ma lettre mais tu comprendras en tous cas mon intention. Présente mes respectueux compliments à tes parents et à ta femme et salue cordialement tous ceux que j'ai connus à Sion, s'ils sont là. Et ton cousin aussi, bien entendu. Les enfants sont moins sensibles aux salutations d'un inconnu qu'à du chocolat.

7 Démissionnaire au Collège classique, Gilliard se voit tout de même proposer par le directeur de celui-ci, Édouard Payot, un statut de maître remplaçant pour l'année scolaire I9II-I9I2, après laquelle, suite au départ de Vallotton, il intègre à nouveau le Gymnase cantonal.

8 Le géographe Élisée Reclus (I830-I905), exilé en Suisse de I872 à I890, a vécu de I879 à I890 à Clarens, près de Montreux. Anarchiste militant, il connaît le père de Roorda, qui partage ses convictions ; ils ont été voisins à Clarens de I88I à I885. Après son départ en France, il a échangé une correspondance avec Roorda. 
Henri Roorda à Marie Gilliard-Malherbe ${ }^{9}$

Square J. Olivier, B

7 septembre I9II

\section{Chère Madame,}

J'ai reçu hier soir les trois volumes de votre aimable lettre. Je suis d'autant plus sensible à votre bonté qu'une personne âgée ne peut pas écrire une longue lettre sans se fatiguer un peu, j'imagine.

Si j'ai été heureux de constater la bonne opinion que vous avez de moi, Chère Madame, ce n'est pas, hélas ! que cette opinion me paraisse tout à fait justifiée ; mais votre lettre contient une expression nouvelle de la bienveillance que vous m’avez déjà témoignée plus d'une fois. La vie est, en réalité, tellement difficile, elle nous menace de tant de façons, qu'on en arrive à accepter avec joie même les approbations et les secours qu'on n'a pas mérités. Le seul fait de la fatigue quotidienne nous empêche souvent d'agir comme nous nous étions promis de le faire. Quant à moi, j'ai reconnu qu'on ne peut pas donner 39 leçons par semaine et conserver en plus beaucoup d'énergie utile. Dans ces fréquents moments de lassitude, on peut bien songer à l'idée qu'on aime et qu'on voudrait répandre ; mais cette idée ne soulève pas, elle ne fait pas agir. On remet l'action désintéressée à plus tard ; et l'on serait tout prêt à accepter, comme le premier venu, une existence tranquille et confortablement bourgeoise.

En définitive, je veux dire, Madame, qu'il doit être bien difficile de mériter les épithètes trop flatteuses de votre lettre. C'est égal : votre jugement erroné m'a rappelé un vieux projet que j'ai fait souvent. Avant de souhaiter la retraite définitive, le repos et un peu de confort, je me dis qu'il faudra absolument écrire un petit livre où je mettrai la plus profonde de mes convictions et où je dirai tout le bien que l'on pourrait faire aux écoliers et qu'on ne leur fait $\mathrm{pas}^{\mathrm{IO}}$.

Veuillez excuser, Madame, ce bavardage : je voulais seulement vous dire que votre lettre ne m'a pas laissé indifférent.

Moi aussi je suis très heureux d'être l'ami d'Edmond. Ce qui me plaît infiniment dans sa conversation, c'est qu'il veut toujours exprimer son opinion sincère ; et qu'il n'use pas de ces formules banales, admises partout, "publiques" en quelque sorte, qu'affectionnent les imbéciles et les lâches. Je me promets d'avoir bientôt avec lui quelques heures de bon bavardage.

9 Marie Gilliard-Malherbe (I848-I9II) est la mère d'Edmond Gilliard. Elle vit à Sion où son mari est à la tête d'un domaine viticole, après avoir tenu seule pendant plusieurs années, jusqu'en 1900, une pension de famille à Lausanne. Elle est l'auteure d'un récit autobiographique publié à titre posthume (Gilliard-Malherbe 20II) Elle mourra à Sion quelques mois après cette lettre, le 29 décembre I9II.

Io Le Pédagogue n'aime pas les enfants, en 1917 , réalisera ce projet. 
Comme vous le dites, Madame, toutes les pages de L'Idéaliste ne sont pas intéressantes ${ }^{\mathrm{II}}$. D'autre part, mon temps étant très limité, je ne lirai pas les deux volumes au complet. (Il ne suffit décidément pas d'avoir des sentiments élevés pour pouvoir écrire un beau livre.) Mais, en feuilletant l'ouvrage, j'y ai déjà trouvé des passages qui m'ont beaucoup plu, un, en particulier, concernant l'éducation.

Ce sera un très vif plaisir pour moi d'aller une fois de plus vous faire une visite à Sion. Merci beaucoup.

Veuillez, Chère Madame, présenter aux vôtres mes compliments les meilleurs et agréer l'expression de mes sentiments très respectueusement dévoués.

Hr. Roorda v. E.

\section{Henri Roorda à Edmond Gilliard}

Mercredi, 23 oct[obre I9I2]

\section{Cher ami,}

Courage! Plonge-toi demain, après-demain et samedi, dans la préparation de tes conférences ${ }^{12}$. C'est encore le travail, avec ses hauts et ses bas, qui est le meilleur remède. (Nom de Dieu ! quel style !) À propos de style, je dois te répéter que ton article sur notre Culture nationale, est excellent ${ }^{13}$. Voilà un jeu qui nous reste dans les jours de spleen et de dèche : ARRANGER DES MOTS! Pour supporter nos mornes journées d'école pensons à cet exercice agréable (et douloureux) qui nous attend.

Et pensons à la raclette de $1913^{14}$. Pour celle de cette année, j'aurais dû emprunter à un ami le prix du chemin de fer. Demain, à $2 \mathrm{~h}$. (ou Ih50 m) je passerai chez toi pour te rendre cent sous. Que cela ne change rien à tes projets.

II Roorda fait très probablement allusion aux Mémoires d'une idéaliste de Malwida von Meysenbug (I8I6-1903), dont le parcours intellectuel et l'engagement féministe a dû intéresser Marie Gilliard-Malherbe. Une édition en deux volumes, préfacée par Gabriel Monod, a paru à Paris, aux Éditions Fischbacher, en I90o.

I2 En novembre et décembre I9I2, Gilliard donnera à l'École Vinet un cycle de cinq cours-conférences intitulé «Études d'histoire poétique vaudoise » et portant sur la poésie vaudoise du XIX siècle. I3 Roorda fait allusion à la chronique intitulée "Culture nationale - Quelques réfléxions sur l'Oberland bernois - F.-A. Forel » que Gilliard a donnée à la Bibliothèque universelle en octobre I9I2 (voir Gilliard I965 : I477-I484).

I4 Edmond Gilliard rappelle dans une « Page retrouvée » : « Nous étions trois ou quatre à avoir lié amitié dans le triste local de la Salle des maîtres - Roorda, Ansermet, Benjamin Grivel et moi -. Nous avions notre raclette d'automne à Sion ; de la pinte du soir au midi du lendemain, en pleine vigne, vin dans l'arrosoir, dans un coin de mur, à feu de sarments » (Gilliard 1965 : I533). 
Ma femme ${ }^{15}$ était hier soir au concert. Une fois de plus elle s'est dit que la tienne a une très belle voix ${ }^{16}$. Elle a donc eu beaucoup de plaisir. Elle n'a pas été dans les "coulisses" pour les félicitations d'usage (qui eussent été sincères) car elle craint les gueules lausannoises dans le goût de celles qu'il y avait dans la salle. Elle en craint beaucoup, de ces gueules, la pauvre!

Mon conseil est sincère, car c'est celui que je dois me donner à moimême : Pense au livre sincère où tu te soulageras.

À demain.

Ton Hr. R.

\title{
Henri Roorda à Edmond Gilliard
}

\author{
Mercredi, 5 h [I9I4]
}

\section{Cher ami,}

S'il fait beau demain nous allons tous à Évian, voir nos sympathiques Français. En cas de pluie, c'est renvoyé à vendredi. S'il pleut encore ce sera samedi. Ou lundi. Donc :

Incertitude.

D'ailleurs, je dois pendant quelques jours m'interdire de nouvelles distractions, de nouveaux plaisirs : je dois me plonger dans ma géométrie ${ }^{17}$ qui ne va pas du tout. Ce matin je l'ai entièrement recommencée. Hier soir, cette perspective de tout recommencer, ce nouvel élan, m'a réconforté. Mais aujourd'hui, devant mon papier, je me décourage déjà un peu. Le courage ne revient que lorsque je ferme mon encrier pour aller prendre mon chapeau. C'est ce que je vais faire.

Quand je saurai quelque chose de sûr quant à notre voyage à Évian, je te reproposerai notre frugal repas du soir.

À bientôt.

Salue cordialement les Ansermet.

Ton

H. R.

I5 La femme de Roorda est Émilie Marguerite Ragozzi dite Lili (I877-1940), qu'il a épousée en 1898 .

I6 Alice Gilliard-Burnand (I876-I943) enseigne le chant au conservatoire de Lausanne depuis 1912. Le concert auquel Roorda fait allusion est un récital qu'elle a donné à la Maison du Peuple le 22 octobre, accompagnée au piano par sa collègue Juliette de Crousaz.

17 Le Manuel de géométrie auquel Roorda travaille paraîtra à Lausanne, chez Payot, en 1915. 


\title{
Henri Roorda à Edmond Gilliard
}

\author{
Square J. Olivier, B
}

[Février I9I4]

\section{Cher Edmond,}

Je viens d'écrire à $\mathrm{May}^{18}$, président de la Commission des manuels, et à mon éditeur que je renonce à ma géométrie pour le moment, - en dépit des promesses formelles que je leur avais faites.

Depuis 3 ans, sauf de rares exceptions, je consacre tous mes jours de vacances et tous mes dimanches à la rédaction de mes manuels. Je suis fatigué et ça ne va plus. Je croyais que durant les 3 jours de congé que le médecin vient de m'octroyer pour pharyngite je pourrais travailler. Mais chaque fois que j'ai voulu me mettre à la besogne le dégoût est venu, le dégoût et l'énervement. J'éprouve très fort le besoin de me taire; de n'avoir aucune tâche pressante; aucun devoir à accomplir.

Je suis sûr que si je me repose 3 ou 4 semaines (un repos relatif, car il me reste mes 35 leçons) la santé et la confiance et l'ardeur reviendront.

La décision que j'ai prise au sujet de ma géométrie peut avoir pour moi des conséquences graves, au point de vue moral et, surtout, au point de vue pécuniaire. Car on comptait sur mon livre. Ce n'est donc pas sans raisons sérieuses que je viens te demander de ne pas compter sur ma collaboration pour le $n^{\circ}$ d'avril $^{19} .20$ fois j'ai pensé à cet article ; et chaque fois mon impuissance m'a énervé.

Si j'ai bien compris, il faut que le $n^{\circ}$ d'avril ait un nombre de pages suffisant. Ne pourriez-vous pas allonger un peu vos articles? Je ne vous demanderais pas d'allonger des sonnets ; mais des considérations générales ! Cornaz, averti, et Cingria ${ }^{20}$, et les autres, ne pourraient-ils pas mettre chacun I page ou 2 de plus ? Ajouter un Carnet ? Il y a encore 6 semaines. Dans 3 ou 4 semaines je serai mieux. Mais est-ce sûr? Crois-moi, Edmond : c'est maintenant que je me dérobe parce que devant mon travail je suis paralysé. Mais si ma défaillance causait un léger dommage aux Cahiers (oh ! très léger !) je saurais plus tard, sois-en sûr, faire mon possible pour le réparer.

Sois généreux ; n'aie aucune rancune. L'idée d'un travail intelligent à faire pour bientôt me met dans un état d'agitation qui m'inquiète. Sois plus

I8 Samuel May (I869-I964), directeur de 1902 à I9I9 du Collège et du Gymnase scientifique, où il continuera d'enseigner comme maître de mathématiques jusqu'en 1935.

I9 Allusion au $2^{\mathrm{e}}$ Cahier vaudois, intitulé « Opinions et rubriques », daté d'avril I9I4; y figurent notamment, outre les contributions d'Alexandre Cingria et de Frédéric Roger-Cornaz mentionnées ensuite, des textes de Gilliard, de Budry et d'Ansermet.

20 Frédéric Roger-Cornaz (1883-1970) et Alexandre Cingria (I879-1945). 
calme que moi et considère que la situation n'a rien de grave. Et malgré tout crois à ma bonne volonté.

Ton Hr. Roorda v. E.

\section{Henri Roorda à Edmond Gilliard}

\section{Cher Edmond,}

Samedi, midi [1914-I9I5]

Grivel $^{21}$ m'a fait ce matin, très discrètement, le reproche que je me suis fait à moi-même plus d'une fois. On a constaté, m'a-t-il dit, que je ne prends pas souvent part aux manifestations des C[ahiers] $v[a u d o i s]^{22}$. Le fait est que dans mon esprit je sépare très nettement ce qui est toi de ce qui est "groupe des C[ahiers] v[audois]"23. Si je pouvais causer avec toi tranquillement, pendant une heure (car la vérité n'est pas simple) je ne parviendrais peut-être pas à te faire admettre le bien-fondé de mes jugements, mais tu ne verrais plus rien d'étonnant dans mon attitude. Mais quand trouverons-nous une heure de "relâche" pour causer de notre vie ? (Â moins qu'une promenade, vers la fin d'un de ces prochains dimanches... ?)

En attendant, avant que des qualificatifs trop sévères te viennent aux lèvres, je te dirai que mon esprit est encombré et que les mouvements de ma pensée ne se font pas avec aisance. Je n'ai pas le temps, ces jours-ci, de mettre de l'ordre en moi-même. Peut-être retrouverai-je plus tard un "moi" plus stable, que les autres pourront en toutes circonstances reconnaître et qui ne trompera pas leurs prévisions.

Consens, cher ami, à attendre. Jusque-là, nous aurons, tous les deux, de quoi nous occuper.

\section{Ton Hr. R.}

2I Benjamin Grivel (1876-I946), ami de Ramuz et de Gilliard, collègue de ce dernier et de Roorda au Gymnase cantonal.

22 Fondés par Edmond Gilliard et Paul Budry, lancés en mars I9I4 par la parution de l'essai inaugural Raison d'être de Ramuz, les Cahiers vaudois joignent à leur vocation éditoriale d'autres activités culturelles; ainsi, en juin 19I4, le «Salon » des Cahiers vaudois, une exposition de peinture, et, régulièrement, des conférences.

23 Rappelons que, avant de publier dans les Cahiers vaudois, en I9I7, Le Pédagogue n'aime pas les enfants, Roorda collaborera en février 1915 au $12^{\mathrm{e}}$ cahier, "Propos de paix et de guerre ", avec «Mon internationalisme sentimental». 


\section{Lili Roorda à Edmond Gilliard}

Lausanne, Io novembre $1925^{24}$

Av. Juste Olivier, 22.

Cher ami,

Votre mot m'a été très précieux, merci. Henri m’a souvent parlé de vous, pour qui il avait beaucoup d'amitié et une très haute estime. Ce que vous direz de lui - quand vous voudrez, sera sûrement bien.

Je vous envoie mes sentiments de reconnaissance.

Lili Roorda van Eysinga

\section{Lili Roorda à Alice Gilliard-Burnand}

Lausanne, 9 décembre 1925.

Av. Juste Olivier, 22

Chère Madame,

Je regrette que vous ayez des malades chez vous ; je l'ignorais, sinon vous pensez bien que je ne vous aurais pas importunée avec mes histoires de dictionnaires.

Pardonnez-moi, je vous prie, et merci du renseignement.

Hier j'ai été mener Miquette ${ }^{25}$ à Leysin à cause de son affreuse glande, j'espère qu'elle se guérira rapidement. Et comme je me sens tout de même un peu seule, je vais m'en aller quelques jours chez Monsé et Berto ${ }^{26}$ qui me réclament depuis longtemps.

Je souhaite à vos malades une prompte guérison et vous prie de croire, chère Madame, à mes sentiments les meilleurs.

Lili Roorda van Eysinga

24 Roorda s'est suicidé trois jours plus tôt, le 7 novembre.

25 Marguerite, la fille cadette des Roorda (1908-1993).

26 Nous n'avons pas pu identifier ce couple d'amis de Lili Roorda. 


\section{Lili Roorda à Edmond Gilliard}

Lausanne, 29 décembre 1925.

Av. Juste Olivier, 22

Cher Monsieur et ami,

Je vous remercie beaucoup de votre envoi ${ }^{27}$, je suis très touchée que vous ayez pensé à moi. Il y a des moments et des circonstances où l'on ressent les choses, bonnes ou mauvaises, plus violemment qu'à l'ordinaire.

Je sais, ayant assisté à vos conférences, que vous abordez des sujets un peu ardus pour la moyenne des intelligences, mais même si votre œuvre est par moments au-dessus de ma pensée, je la lirai sûrement avec intérêt.

Veuillez transmettre, je vous prie, mes meilleurs messages à Madame Gilliard et croire à mon affectueuse reconnaissance

Lili Roorda van Eysinga

\section{Lili Roorda à Edmond Gilliard}

Lausanne, 27 novembre 1926. 22, Av. Juste Olivier.

\section{Cher Monsieur et ami,}

Pardonnez-moi d'avoir tardé à vous remercier : j'ai été malade toute la semaine. Lundi j'étais au lit, incapable de me tenir debout, c'est pourquoi je n’ai pu vous entendre, mais je sais que votre conférence a été admirable ; si, par hasard, elle est écrite, je serais heureuse de la lire ${ }^{28}$.

Je suis contente qu'avec l'autorité de votre grand talent, vous ayez tenu à rendre justice à un homme envers qui, sous prétexte de sa fin malheureuse, beaucoup se sont empressés d'être injustes. Car s'il fut un fichu mari cela ne regarde que moi et cela ne l'a pas empêché d'être remarquable comme pédagogue, écrivain et conférencier.

Merci encore, cher Monsieur et croyez-moi votre bien reconnaissante

$$
\text { E. L. Roorda van Eysinga }
$$

27 Gilliard vient de faire paraître en octobre, aux Éditions Payot, son premier livre, regroupant plusieurs essais et conférences, sous le titre Rousseau et Vinet individus sociaux.

28 Lundi 22 novembre 1926, à la Maison du Peuple à Lausanne, Gilliard a donné une conférence sur Roorda, intitulée « Henri Roorda ou le pédagogue contre l'enfant ». 


\section{Lili Roorda à Edmond Gilliard}

Lausanne, 4 novembre 1929. 22, Av. Juste Olivier.

\section{Cher Monsieur,}

Je reçois à l'instant votre aimable billet. Je serai très heureuse de vous voir et vous remercie d'avance de la plaquette que vous voulez bien me donner ; je suis sûre que ce sera parfait ${ }^{29}$. Puis-je vous demander de me téléphoner (27942) avant de venir ici, car je désire ne pas manquer votre visite.

J'ai des excuses à vous faire, elles viennent un peu tardivement : il y a quelques mois, je vous ai rencontré à l'Avenue d'Ouchy ; peut-être vous seriez-vous arrêté un instant, j'en ai eu ensuite l'impression, mais sans doute mon air pressé et sauvage vous a étonné, cela tenait à ce que, n'étant pas sortie pendant deux ans, j'étais tout à fait étourdie et voulais rentrer chez moi au plus vite.

Recevez, cher Monsieur, l'expression de mes sentiments les meilleurs.

$$
\text { E. L. Roorda van Eysinga }
$$

\section{Bibliographie}

Gilliard, Edmond, Euvres complètes, Genève, Trois Collines, 1965.

Gilliard-Malherbe, Marie, À létroit dans ma peau de femme, Lausanne, En bas, 2011.

Ramuz, C. F., Journal, vol. 2, Euvres complètes, Genève, Slatkine, 2005, t. II.

29 À Henri Roorda, de Gilliard, vient de paraître aux Éditions des Petites Lettres de Lausanne (série I, $\mathrm{n}^{\circ} 4$, daté d'octobre 1926). 